\title{
Distrofia macular cristalina em paciente com síndrome de Sjögren-Larsson: relato de caso
}

\author{
Macular crystalline dystrophy in Sjögren-Larsson syndrome: case report
}

\author{
David Leonardo Cruvinel Isaac ${ }^{1}$ \\ Gustavo Henrique Medeiros Queiroz ${ }^{2}$ \\ Caroline Campelo Feres ${ }^{3}$ \\ Marcos Ávila ${ }^{4}$
}

Trabalho realizado no Centro de Referência de Oftalmologia (CEROF) da Universidade Federal de Goiânia UFG - Goiânia (GO) - Brasil.

${ }^{1}$ Doutor, Médico Assistente e Coordenador do Setor de Retina e Vítreo do Centro de Referência de Oftalmologia (CEROF) da Universidade Federal de Goiás - UFG - Goiânia (GO) - Brasil.

${ }^{2}$ Residente do CEROF da UFG - Goiânia (GO) - Brasil. ${ }^{3}$ Residente em Fisiatria do Centro de Reabilitação e Readaptação Dr. Henrique Santillo - CRER - Goiânia (GO) - Brasil.

${ }^{4}$ Doutor, Professor Adjunto e Chefe do Serviço de Oftalmologia do CEROF da UFG - Goiânia (GO) - Brasil.

Endereço para correspondência: David L. C. Isaac.

Rua C-259, 441 - Setor Nova Suíça - Goiânia (GO)

CEP 74280-220

E-mail: cruvinelisaac@hotmail.com

Recebido para publicação em 13.06.2008

Aprovação em 20.12.2008

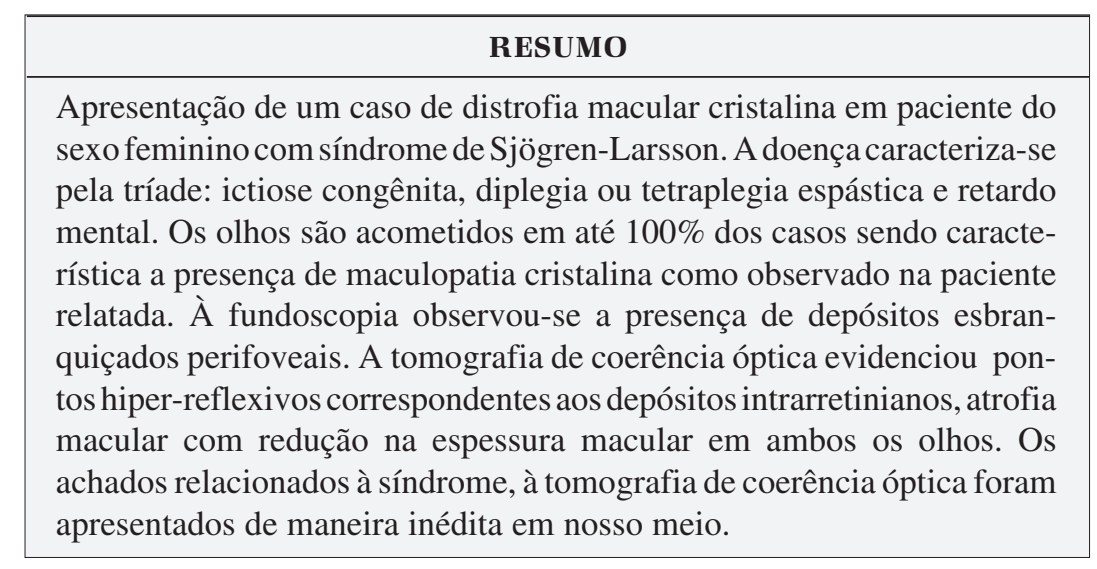

Descritores: Macula lútea/patologia; Síndrome de Sjögren-Larsson/complicações; Tomografia de coerência óptica/métodos; Relatos de casos [Tipo de publicação]

\section{INTRODUÇ̃̃̃O}

A síndrome de Sjögren-Larsson (SSL) constitui uma doença geneticamente herdada e caracterizada pela tríade: ictiose congênita, diplegia ou tetraplegia espástica e retardo mental ${ }^{(1)}$. Descrita inicialmente em $1957^{(1)}$, apresenta transmissão por herança autossômica recessiva e sua prevalência estimada é menor que 0,4 por 100.000 habitantes ${ }^{(2)}$. A síndrome é causada por um erro inato no metabolismo dos lipídios pela deficiência da enzima microssômica aldeído graxo desidrogenase. Esta deficiência leva à deposição de metabólitos lipídicos nos tecidos, sendo esta considerada a principal causa dos sintomas relacionados à síndrome ${ }^{(3)}$.

Além de alterações cutâneas e neurológicas, grande parte dos pacientes acometidos pela SSL apresenta alterações visuais como fotofobia e diminuição da acuidade visual, relatados desde os primeiros anos de vida. Nestes pacientes descreve-se a presença de maculopatia cristalina, caracterizada pela presença de pontos esbranquiçados dispostos circunjacentes à fóvea, em até $100 \%$ dos $\operatorname{casos}^{(4)}$. Em 1987, Nilsson e Jagell ${ }^{(5)}$, descreveram pela primeira vez o aspecto anatomopatológico da mácula de um paciente portador da SSL. Em seu relato demonstrou-se a preservação da arquitetura celular foveal e descreveu-se a presença de grânulos de lipofuscina intrarretinianos, possivelmente responsáveis pelos pontos esbranquiçados observados oftalmoscopicamente. Recentemente dois estudos analisaram o aspecto foveal de pacientes com SSL pelo tomógrafo de coerência óptica $(\mathrm{OCT})^{(6-7)}$. Nos dois estudos encontraram-se alterações pelo OCT tendo sido demonstrada alterações como a presença de pontos hiper-reflexivos ao redor da fóvea, atrofia foveal generalizada e presença de cistos no centro da fóvea. 
No presente trabalho relatamos o caso de uma paciente portadora de síndrome de Sjögren-Larsson e distrofia macular cristalina, descrevendo os achados fundoscópicos e aspectos à tomografia de coerência óptica da maculopatia. Este relato é o primeiro a descrever os achados clínicos e ao OCT envolvendo a síndrome em nosso meio.

\section{RELATO DE CASO}

T.S.S., sexo feminino, cor parda, 13 anos de idade, encaminhada por serviço de genética e reabilitação com diagnóstico genético de síndrome de Sjögren-Larsson para avaliação oftalmológica. A mãe referia que a adolescente apresentava dificuldades visuais desde a infância além de importante fotofobia. Relatava consanguinidade (pais primos em primeiro grau), tendo a mãe apresentado 5 gestações com 3 irmãos presumidamente normais e 1 natimorto. Refere ter nascida prematura aos 8 meses de gestação (não sabendo informar o tempo gestacional em semanas) e que havia 1 tia materna com quadro semelhante, já falecida. Negava outras doenças ou utilização de medicações. Ao exame físico apresentava fácies alegre e pouco comunicativa, pele mostrando ressecada e com descamação (ictiose), marcha com padrão diparético, em tesoura, com flexão de quadril e joelhos em todas as fases da marcha, apoio com pé em equino e desabamento do arco plantar. Apresentava sinais nítidos de retardo mental com dificuldades de compreensão de perguntas elaboradas e resposta com palavras curtas. Ao exame oftalmológico apresentava melhor acuidade visual corrigida de 20/60 no olho direito (OD) e 20/70 no olho esquerdo (OE) (com o E de Snellen), apresentando equivalente esférico de $-3,00 \mathrm{em}$ ambos os olhos (AO). Apresentava exame biomicroscópico normal e
PIO de $14 \mathrm{mmHg}$ em AO. Ao exame de oftalmoscopia indireta e biomicroscopia de fundo foram observados depósitos intrarretinianos puntiformes e arredondados, de coloração esbranquiçada ao redor da fóvea. O centro foveal não apresentava sinais oftalmoscópicos de acometimento (Figura 1). À angiofluoresceinografia (Imagenet 2000, Topcon Corporation, Tóquio, Japão) observou-se, em ambos os olhos, discreto padrão de fluorescência transmitida em toda fóvea sem sinais, no entanto, de vazamentos ou impregnação tardia (Figura 2). A tomografia de coerência óptica (STRATUS OCT, software 4.0.5, Carl Zeiss Meditech, Dublin, Estados Unidos) evidenciou aumento da reflexividade na região perifoveolar correspondente aos pontos intrarretinianos além de espessura macular central de $121 \mu \mathrm{m}$ no olho direito e $119 \mu \mathrm{m}$ no olho esquerdo (Quadro 1).

\section{COMENTÁRIOS}

A síndrome de Sjögren-Larsson (SSL) constitui doença rara, herdada por herança autossômica recessiva e que apresenta acometimento sistêmico. A despeito das clássicas alterações clínicas que compõem a tríade da doença (ictiose congênita, espasticidade e retardo mental), sintomas oculares e achados fundoscópicos são igualmente prevalentes. Inicialmente, há 50 anos, a maculopatia cristalina foi descrita como presente em 10,7\% dos pacientes com a doença ${ }^{(1)}$, no entanto, estudos recentes demonstraram o acometimento de $100 \%$ dos pacientes acometidos com a síndrome, além de sintomas como fotofobia e redução da acuidade visual ${ }^{(2,7-9)}$.

O exame de tomografia de coerência óptica (OCT) foi empregado recentemente em pacientes com SSL. Dois estudos descreveram as características maculares de pacientes com a síndrome, sendo descritas alterações ao OCT em todos os pa-
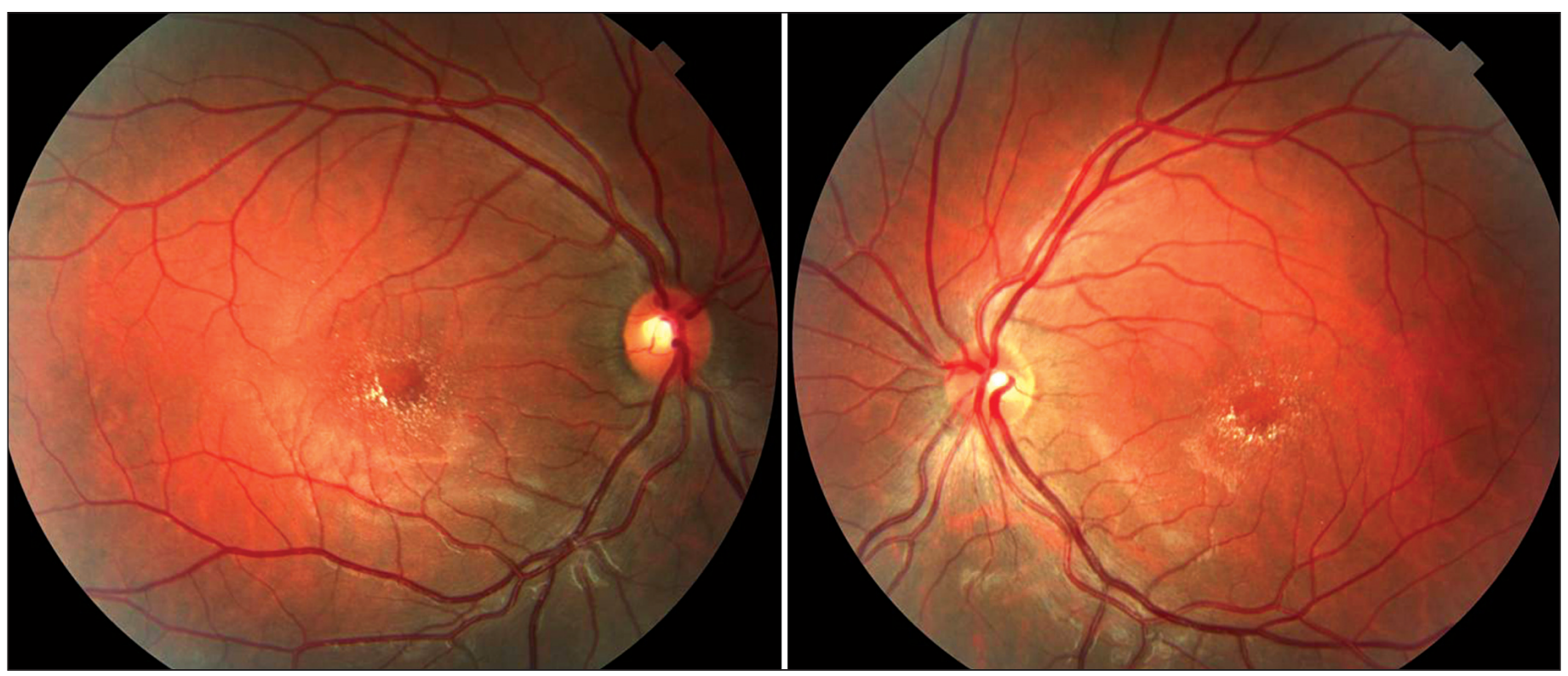

Figura 1 - Retinografia colorida mostrando o padrão dos depósitos cristalinos ao redor da fóvea 

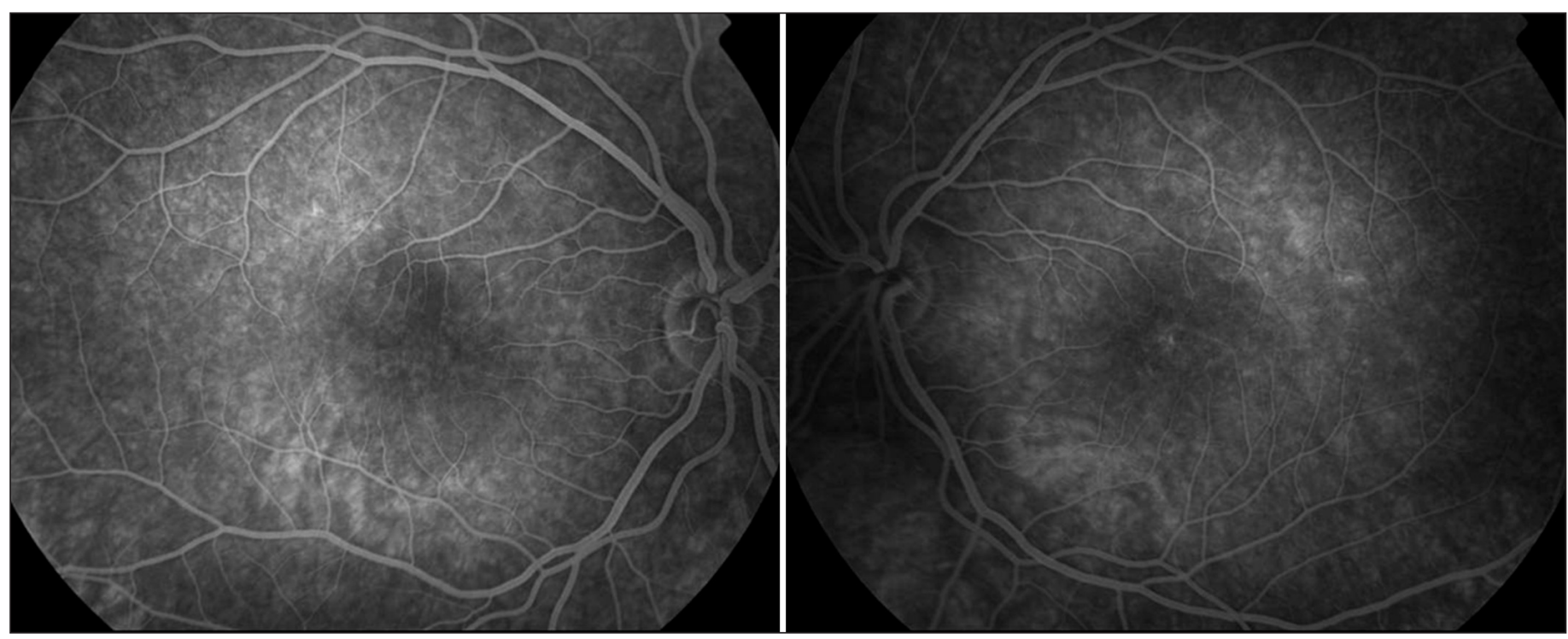

Figura 2 - Angiofluoresceinografia mostrando despigmentação macular e discreta fluorescência transmitida
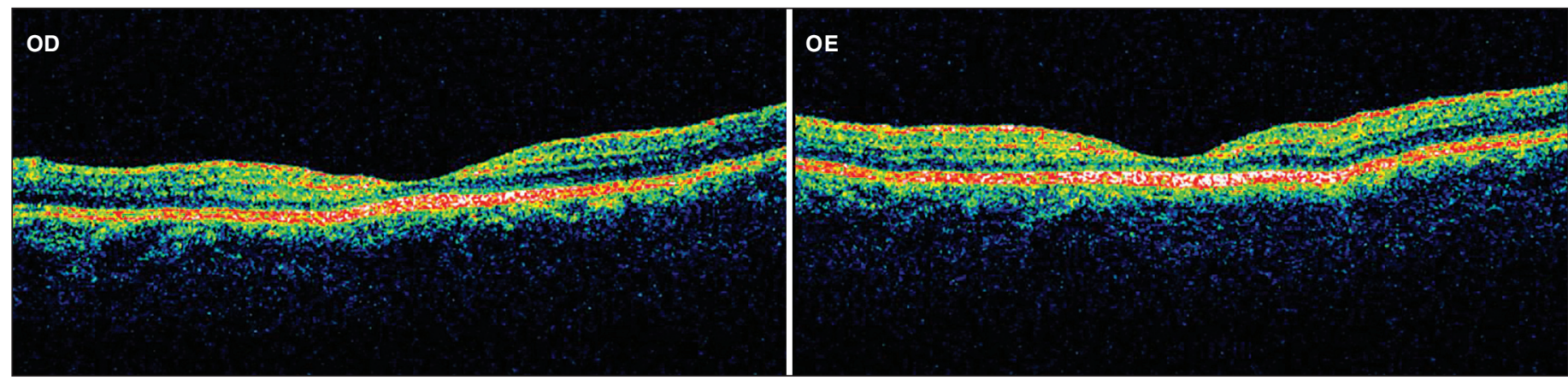

Quadro 1. Tomografia de coerência óptica mostrando importante afilamento da mácula em ambos os olhos com visível diminuição da espessura da camada nuclear externa e aumento da reflexividade perifoveolar

cientes estudados ${ }^{(7,9)}$. As alterações descritas mais frequentes são o achado de pontos hiper-reflexivos na região perifoveolar, afilamento macular generalizado e cistos intrarretinianos foveais. No presente caso o OCT demonstrou, em ambos os olhos, a presença de regiões hiper-reflexivas nas regiões correspondentes à camada de fibras nervosas e plexiforme interna. Observou-se ainda, importante afilamento macular difuso com particular redução da espessura da região correspondente aos segmentos externos dos fotorreceptores e à camada nuclear externa, se comparados à anatomia normal macular ao OCT e valores de espessura central da fóvea ${ }^{(8)}$ (Quadro 1).

Nilsson e Jagell ${ }^{(5)}$ estudaram a anatomia macular de paciente falecido com SSL. Não observou-se alterações anatômicas na fóvea, porém notou-se a presença de grânulos de lipofuscina nas células do epitélio pigmentado da retina com consequente diminuição da quantidade de melanina, condição que poderia explicar a observação de leve defeito em janela à angiofluoresceinografia no presente caso. Em sua análise histopatológica os autores não descreveram inclusões intrarreti- nianas, como parece ocorrer à observação fundoscópica e ao OCT neste e em outros estudos ${ }^{(6-7,9)}$.

A síndrome de Sjögren-Larsson é doença hereditária e rara caracterizada pela tríade: ictiose congênita, espasticidade e retardo mental. O presente estudo relatou achados retinianos relacionados à síndrome, como a maculopatia cristalina e atrofia macular ao OCT, descritos pela primeira vez em nosso país. A maculopatia cristalina relacionada à doença foi observada em todos os pacientes de relatos recentes publicados em literatura indexada, submetidos a exame fundoscópico e ao OCT. Novos trabalhos com maior número de casos são necessários para uma afirmação categórica, no entanto a universalidade da maculopatia cristalina até o momento em pacientes com SSL permitiria incluí-la em uma nova tétrade diagnóstica para a doença.

\section{ABSTRACT}

Presentation of a case of crystalline macular distrophy diagnosed in a female patient with Sjögren-Larsson syndrome. 
The disease consists of clinical findings of spastic diplegia or tetraplegia, mental retardation, and congential ichthyosis. The eyes are affected in up to $100 \%$ of cases, and crystalline maculopathy is the main finding as described in this case report. On fundus examination multiple white dots were observed at ophthalmoscopy. The optical coherence tomography has shown not only the hipereflexive intraretinal spots but also macular atrophy with macular thickness reduction. The tomographic findings were first described in our country.

Keywords: Macula lutea/pathology; Sjögren-Larsson syndrome/complications; Tomography, optical coherence/methods; Case reports [Publication Type]

\section{REFERÊNCIAS}

1. Sjögren T, Larsson T. Oligophrenia in combination with congenital ichthyosis and spastic disorders; a clinical and genetic study. Acta Psychiatr Neurol Scand Suppl. 1957;113:1-112.
2. Willemsen MA, IJIst L, Steijlen PM, Rotteveel JJ, de Jong JG, van Domburg $\mathrm{PH}$, et al. Clinical, biochemical and molecular genetic characteristics of 19 patients with the Sjögren-Larsson syndrome. Brain. 2001;124(Pt 7):1426-37.

3. Rizzo WB. Sjögren-Larsson syndrome: fatty aldehyde dehydrogenase deficiency. In: Scriver CR, Beaudet AL, Sly WS, editors. The metabolic and molecular bases of inherited disease. $8^{\text {th }}$ ed. New York: McGraw-Hill; 2001. v.2. p.2239-58.

4. Willemsen MA, Cruysberg JR, Rotteveel JJ, Aandekerk AL, Van Domburg PH, Deutman AF. Juvenile macular dystrophy associated with deficient activity of fatty aldehyde dehydrogenase in Sjögren-Larsson syndrome. Am J Ophthalmol. 2000;130(6):782-9.

5. Nilsson SE, Jagell S. Lipofuscin and melanin content of the retinal pigment epithelium in a case of Sjögren-Larsson syndrome. Br J Ophthalmol. 1987; 71(3):224-6

6. Jean-François E, Low JY, Gonzales CR, Sarraf D. Sjögren-Larsson syndrome and crystalline maculopathy associated with a novel mutation. Arch Ophthalmol. 2007; 125(11):1582-3.

7. Fuijkschot J, Cruysberg JR, Willemsen MA, Keunen JE, Theelen T. Subclinical changes in the juvenile crystalline macular dystrophy in Sjögren-Larsson syndrome detected by optical coherence tomography. Ophthalmology. 2008; 115(5):870-5.

8. Chan A, Duker JS, Ko TH, Fujimoto JG, Schuman JS. Normal macular thickness measurements in healthy eyes using Stratus optical coherence tomography. Arch Ophthalmol. 2006;124(2):193-8.

9. Jagell S, Heijbel J. Sjögren-Larsson syndrome: physical and neurological features. A survey of 35 patients. Helv Paediatr Acta. 1982;37(6):519-30. 